\section{To THE EDITOR}

Dr. Alastair Buchan's eloquent editorial on our review of education in the clinical neurosciences (Can J Neurol Sci 2001; 28:281-282) missed the point. Resident training program committees are not bureaucratic regulatory bodies, and do not set call-schedule, morning-after or any other type of mandatory rules. They are committees to oversee and administrate training programs, and among other things ensure their adherence to contracts and guidelines set by governments and the Royal College. If they don't do a reasonable job of that, their programs will not be accredited and will ultimately be suspended. Our review pointed out that it has become impossible for some, and perhaps many, programs to meet these requirements due to diminishing resident numbers and increasingly demanding clinical teaching units - Dr. Buchan himself refers to modern stroke care as "extreme neurology"! One solution that Dr. Buchan took exception to is "hospitalists" to complement the resident staff. Since that job includes night time in-hospital call, it has been difficult to attract Canadian-trained doctors to such positions. With special approval from our provincial College we, in Edmonton, have been able to acquire the services of foreign medical graduates, otherwise unqualified to practice private medicine in Canada, following a period of supervised training. These doctors are hired (by the hospital) precisely to "keep Canadian residents in their beds" as Dr. Buchan worries. While they are fine doctors, they are not, as Dr. Buchan mistakes, foreign neurologists, neurosurgeons or neuroscientists seeking to "light their torches at the fire of the Ancients". There are, in fact, no work regulations regarding international fellows on our services, and thank goodness for that, since all of them are on the stroke team!

We are sorry that Dr. Buchan, and perhaps others, found our review "depressing reading". The constant lament that "things are not what they used to be" may, in the case of medical specialty training today, actually be true for a change. Recognizing this, and dealing with it proactively and effectively, is the progressive approach, not going "back to the future", as Dr. Buchan would suggest.

J. Max Findlay Edmonton, Alberta

\title{
Notes and Announcements
}

\section{Neuroscience Conference in Banff}

The Department of Clinical Neurosciences, University of Calgary, will hold its 9th Annual Banff Conference at the Canmore Radisson Hotel and Conference Centre, near Banff. The Conference will run from Friday, March 15, 2002 to Sunday, March 17 2002. There will be three invited guest speakers from Canada, the United States and the United Kingdom. Topics include spinal cord repair, MRI and multiple sclerosis, and hydrocephalus. There will be case presentations and research presentations from the Department of Clinical Neurosciences, University of Calgary. All neurologists, neurosurgeons, physiatrists, and other interested physicians and researchers are invited.

For more information please contact Ms Danielle Sikander at 403-670-1260 or e-mail at sikander@ucalgary.ca.

\section{Erratum}

Methodology for the Canadian Activase for Stroke Effectiveness Study (CASES). Can. J. Neurol. Sci. 2001; 28: 232-238.

Two members of the CASES Investigators were mistakenly omitted from the CASES Investigators list. They are: Vance Makin (Lions Gate Hospital, North Vancouver), and JH Warwick Pexman (Foothills Hospital, Calgary). 